\title{
Chelation and solvent effect on the preparation of titania colloids
}

\author{
Hung-Jen Chen ${ }^{\text {a }}$, Leeyih Wang ${ }^{\text {b,c }}$, Wen-Yen Chiu ${ }^{\mathrm{a}, \mathrm{b}, *}$

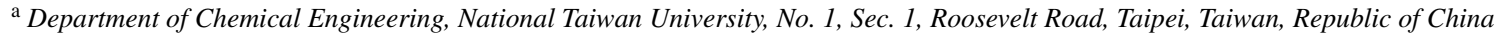 \\ ${ }^{\mathrm{b}}$ Institute of Polymer Science and Engineering, National Taiwan University, Taipei, Taiwan, Republic of China \\ ${ }^{\mathrm{c}}$ Center for Condensed Matter Sciences, National Taiwan University, Taipei, Taiwan, Republic of China \\ Received 13 September 2005; received in revised form 6 January 2006; accepted 4 February 2006
}

\begin{abstract}
Different particle size and stability of $\mathrm{TiO}_{2}$ colloids, using acetylacetone modified titanium isopropoxide as precursor, have been synthesized under various polarity organic solvent. In this study, the chelating bond between titanium isopropoxide and acetylacetone was examined by FTIR. The crystalline structure of $\mathrm{TiO}_{2}$ colloid prepared by using acetylacetone modified or un-modified titanium isopropoxide as precursor were determined by X-ray diffraction, before or after sintering process. The $\mathrm{TiO}_{2}$ colloidal size and aggregation phenomenon were obtained from light scattering and photographs of SEM and TEM. From the observation of experiments, stable $\mathrm{TiO}_{2}$ colloid could not be obtained if the hydrolysis/condensation reaction rate of titanium precursor was not inhibited by acetylacetone. The solvent effect on the colloidal particle size and stability were explained by Hansen solubility parameters. In this study, the Hansen solubility parameters of acetylacetone modified $\mathrm{TiO}_{2}$ were estimated, which would be useful information in the preparation of stable $\mathrm{TiO}_{2}$ colloid.
\end{abstract}

(c) 2006 Elsevier B.V. All rights reserved.

Keywords: Titania colloid; Solvent effect; Hansen solubility parameter

\section{Introduction}

Titanium oxide, a well known catalyst possessing features of chemical/photocorrosion stability, non-toxicity, low cost, and highest oxidation rate among many active metal oxides studied, has been widely used in many application, such as photocatalysts, solar cell, paint, pigment, cosmetics, biomedicine and ceramics [1-14]. In chemical industry, olefin epoxidation, isomerization, alkylation, oxidation of $\mathrm{H}_{2} \mathrm{~S}$ to $\mathrm{SO}_{2}$, and noble metal $(\mathrm{Pt}, \mathrm{Rh}$ or $\mathrm{Ru}$ ) impregnated titania for Fischer-Tropsch synthesis all need titanium oxide for catalysis [15-18]. In biomedical materials, the interaction mechanism of $\mathrm{TiO}_{2}$ and amino acid provides important information in determining the biocompatibility of Ti and Ti alloys with human body, which are widely applied in prostheses materials and medical implant $[19,20]$. In cosmetics, micronised titanium oxide shows increasing importance in the application of skin protection against harmful UV because of its high ability of absorption over broad UV range. In paint and pigment, actually, the earliest role of $\mathrm{TiO}_{2}$ was served

\footnotetext{
* Corresponding author. Tel.: +886 2 23623259; fax: +886 223623259

E-mail address: ycchiu@ntu.edu.tw (W.-Y. Chiu).
}

as white pigment and paint due to its stability to photocorrosion and low cost.

In such many applications, whether the titanium oxide can bring its performance to full play or not depends on the dispersion stability of $\mathrm{TiO}_{2}$ particles in the medium. For example, the efficiency of photocatalysis will be developed to the most degree with largest active surface when the photocatalyst can be dispersed in nano-sized scale and remain its stability. A betterdispersed pigment can show stronger color strength so that the quality of product is improved.

In order to provide the particle stability, lots of efforts have been made on the surface treatment of $\mathrm{TiO}_{2}$ particles and forming hybrid. In the electrostatic stabilizing method, titanium oxide will be stored in aqueous solution stably if the solution was added in nitric acid [21]. Except for electrostatic stabilizing method, ionizable polymers are used for titanium oxide dispersant more and more frequently because they can provide a high charge repulsion and useful steric hindrance.

For polyelectrolyte, such as poly (acrylic acid) (PAA), the partial ionization of the $\mathrm{COOH}$ groups in oligomeric or polymeric acids allows for an acid base or hydrogen bonding interaction with other metal oxide surface, which results in adsorption on the surface at high $\mathrm{pH}[22,23]$. Kuo et al. [6] used the 
Table 1

Composition and appearance of the prepared titania colloids

\begin{tabular}{lllll}
\hline Sample & Solvent & Acac/TIP (mol ratio) & Water/TIP (mol ratio) & Observation \\
\hline Col-THF & Tetrahydrofuran & 2.86 & 4.14 & Clear solution \\
Col-ace & Acetone & 2.86 & 4.14 & Clear solution \\
Col-but & Butanol & 2.86 & 4.14 & Clear solution \\
Col-chl & Chloroform & 2.86 & 4.14 & Turbid solution \\
Col-tol & Toluene & 2.86 & 4.14 & Precipitated solution \\
Col-hex & Hexane & 2.86 & 4.14 & Precipitated solution \\
\hline
\end{tabular}

poly(methylacrylate-acrylic acid) copolymer as the dispersant for dispersing $\mathrm{TiO}_{2}$ in aqueous solution and resulted in low viscosity, thus less sedimentation and more homogenous distribution.

The principle of the methods for stabilizing the $\mathrm{TiO}_{2}$ dispersions mentioned above emphasizes force balance between particles. Another kind of method to prepare stable dispersion is focused on the interaction between particles and solvent. Colvin and co-workers synthesized nanocrystalline titania in nonhydrolytic solution, which resulted in nanocrystals without hydroxyl group on the surface and highly dispersible in organic solvent [24].

In order to understand the force balance in the dispersion system, some theories were proposed to deal with it. The Derjaguin, Landau, Verwey and Overbeek (DLVO) theory [25] combines the attractive van der Waals force and repulsive electrostatic force as a function of the particle distance in suspension and summarizes that a maximum free energy functions as a barrier and keeps the particles away. In another way, the Hansen solubility parameter is frequently used for the theoretical description of stability between $\mathrm{TiO}_{2}$ particles and solvents from the viewpoint of interaction. The interaction of particle and solvent plays a key role in the preparation of stable colloid. Hence, selecting a suitable solvent possessing high interaction with $\mathrm{TiO}_{2}$ particles is a simple and useful method.

In this work, Hansen solubility parameters are applied to investigate how solvent influences the dispersion stability of $\mathrm{TiO}_{2}$ particles

\section{Experiment}

\subsection{Materials}

Titanium (IV) isopropoxide (TIP, $>98 \%$ ), acetylacetone (acac, $>99 \%$ ), 1butanol $(>99.5 \%)$ and toluene $(>99.5 \%)$ were purchased from Acros. Tetrahydrofuran (THF, 99.5\%) acetone (99.5\%), chloroform (99.8\%) and hexane $(99.8 \%)$ were purchased from Mallinckrodt. All these chemicals were used as received. The water used in the sol-gel reaction was purified using the Millipore Milli-Q system.

\subsection{Chelation}

Table 1 shows the reagents and their amount used in the chelation and preparation of $\mathrm{TiO}_{2}$ colloid. The notation was explained by selecting "Col-ace" as an example. Col represented the colloid and ace was acetone. The only experimental variable, solvent, was named by the first three letters for a simple notation Except for Col-THF, it was taken from the generally called name, THF.

A $100 \mathrm{ml}$ round-bottom flask equipped with stirrer, thermometer with a temperature controller and a condenser was used. In a typical example, $2 \mathrm{ml}$ of
TIP was fully dissolved in a premixed solution consisting of $25 \mathrm{ml}$ of different organic solvent and $2 \mathrm{ml}$ of acetylacetone. As the TIP was added into the acac solution, chelation, an exothermic reaction, occurred and yielded a yellow solution.

For a comparative experiment, the TIP was directly dissolved in the organic solvent without chelation with acac. This was for the study of effect of chelation on the preparation of titnaia colloid. The other procedures of colloid preparation were the same as those with chelated TIP.

\subsection{Sol-gel reaction}

After $30 \mathrm{~min}, 0.5 \mathrm{ml}$ of water was added dropwise to the TIP solution and stirred vigorously to avoid local aggregation. The sol-gel reaction was carried out first at room temperature for $30 \mathrm{~min}$. The solution was then heated at $90{ }^{\circ} \mathrm{C}$ for $10 \mathrm{~h}$ for high boiling point solvent, such as butanol and toluene, and other solvents were heated at their boiling point for $10 \mathrm{~h}$. Fig. 1 presents the synthetic route. The different organic solvents were used to analyze their influence on the particles size and dispersion ability of titania in organic solvent.

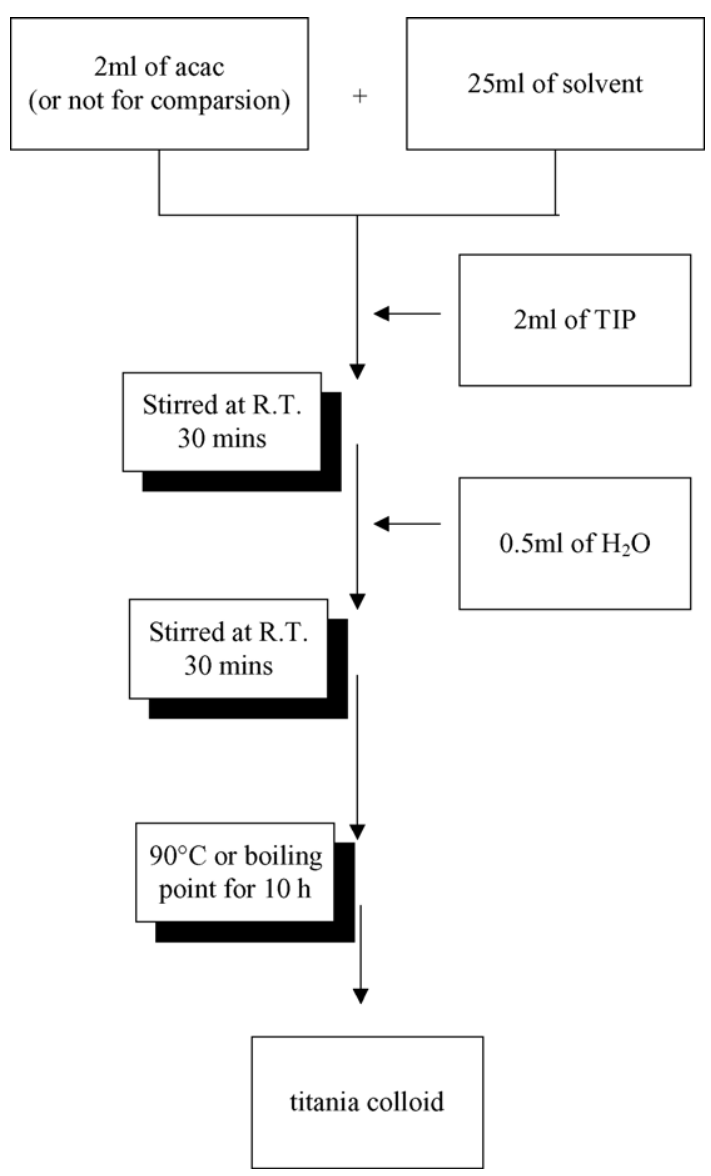

Fig. 1. Synthetic route for the preparation of titania colloid. 


\subsection{Sintering}

The as-prepared titania colloids were dried to form $\mathrm{TiO}_{2}$ powder. Then, the $\mathrm{TiO}_{2}$ powder was sintered at $450{ }^{\circ} \mathrm{C}$ for $1 \mathrm{~h}$ with a heating rate of $5^{\circ} \mathrm{C} \mathrm{min}-1$ from room temperature.

\subsection{Measurements}

The functional groups and chelating behaviors of acac modified TIP were analyzed by a Fourier Transform Infrared spectrophotometer (FTIR) (Bio-Rad, FTS-3000). The sample was prepared by mixing and grinding the dried $\mathrm{TiO}_{2}$ powder with potassium bromide $(\mathrm{KBr})$ and compressed into a pellet. The absorption spectra were recorded with 16 scans at a resolution of $4 \mathrm{~cm}^{-1}$ between $4000 \mathrm{~cm}^{-1}$ and $400 \mathrm{~cm}^{-1}$. X-ray diffraction study (MAC Science Diffractometer MXP-3), using $\mathrm{Cu} \mathrm{K \alpha}$ radiation at $40 \mathrm{KV}$ and $30 \mathrm{~mA}$, was conducted to examine the crystalline patterns of as-prepared and sintered titania. The average particle size of titania colloids was characterized by using laser light scattering (Zeta Sizer 3000H, Malvern). Each colloid was measured three times to make sure of the reproducibility of the data. However, for the colloids with precipitation, only the upper part of solution was taken for light scattering measurement. Therefore, the data obtained represented the average size of the smallest particles of all in the wide range of size distribution. The aggregation phenomena of titania colloids were observed by Hitachi (S-800) Scanning Electronic Microscope (SEM) and JEOL JSM-1200 EX II Transmission Electron Microscope (TEM).

\section{Results and discussions}

\subsection{FTIR analysis}

The reactivity of titanium precursor was known as very high. In the comparative experiment, all the sol-gel reaction of TIP in different organic solvents used here led to obviously immediate precipitation. Acetylacetone (acac) was usually used to control and suppress its hydrolysis reaction rate. The FTIR spectra of acac and titania colloid prepared from different organic solvent were shown in Fig. 2. Acac shows two tautomeric form: one is enol form dominated in most organic solvent and the other is keto form dominated in water, as represented in the following $[26,27]$.

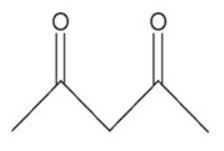

(keto)

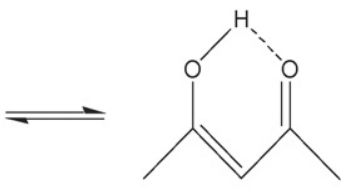

(enol)
Among them, the enol form of acac possesses higher stability, which can be related to the resonance delocalization of the conjugated double bonds through hydrogen bonding [28]. It appeared that the $\mathrm{C}=\mathrm{O}$ vibration of enol tautomeric acac exhibited at $1620 \mathrm{~cm}^{-1}$ while the doublet peaks at $1708 \mathrm{~cm}^{-1}$ and $1728 \mathrm{~cm}^{-1}$ were assigned to the bending vibration of keto tautomeric $\mathrm{C}=\mathrm{O}$ group [29]. When the acac reacted with TIP, these three carbonyl vibration disappeared and two new bands were generated [30]. These two bands from $1580 \mathrm{~cm}^{-1}$ to $1530 \mathrm{~cm}^{-1}$ came from the split of enol form of $\mathrm{C}=\mathrm{O}$ at $1620 \mathrm{~cm}^{-1}$. This indicated the chelation occurring between acac and TIP. The chelation between acac and TIP could be described in the following scheme, which was formed by reacting TIP with $2 \mathrm{~mol}$

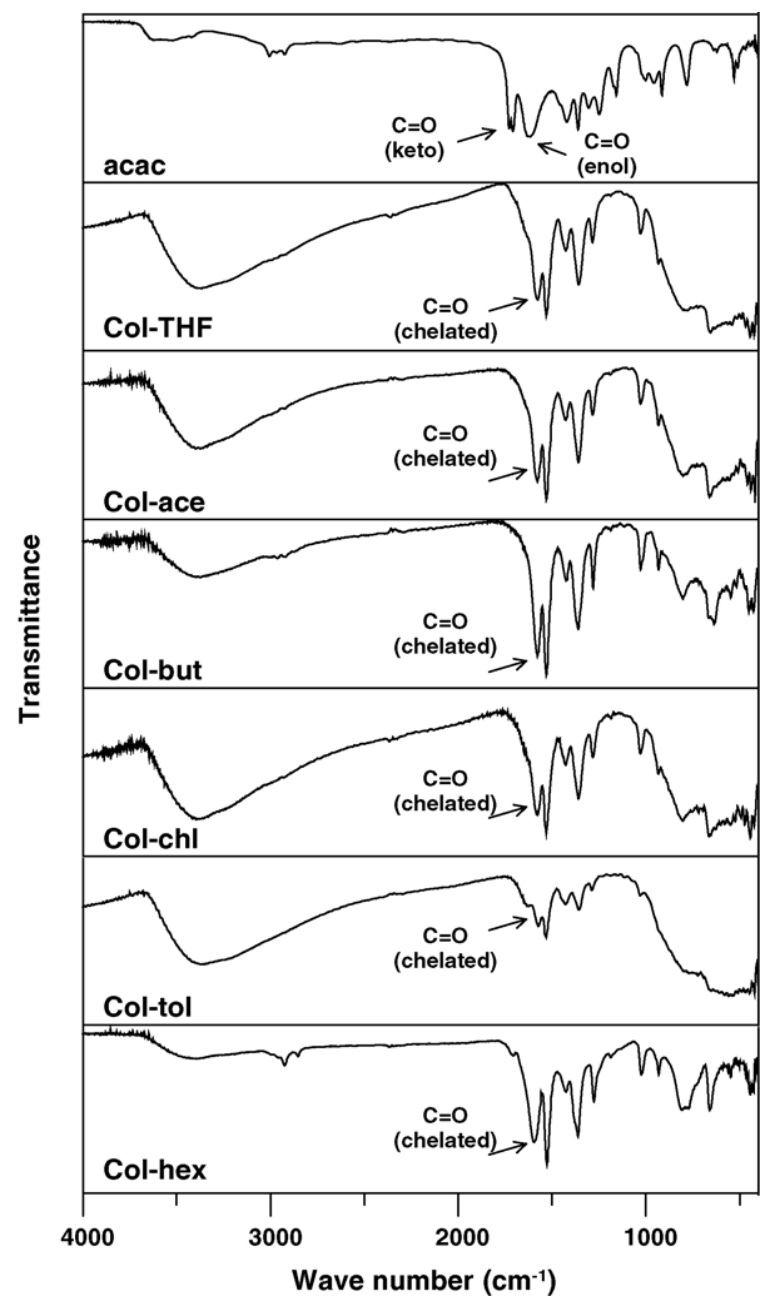

Fig. 2. FTIR spectra of acetylacetone and titania colloid prepared by acac modified TIP.

of acac as an example.<smiles></smiles>

In addition, no bands appear around $1708 \mathrm{~cm}^{-1}$. Haile et al. indicated that the bands at $1700 \mathrm{~cm}^{-1}$ and $1720 \mathrm{~cm}^{-1}$ appeared when the mole ratio of acac to TIP was higher than 3, which suggested that the presence of excess acac after chelation [31]. In addition, they found that if the mole ratio of acac to TIP was less than 2 , the titanium precursor was not fully stabilized. In our study, the mole ratio of acac to TIP was 2.86. No free carbonyl group $(\mathrm{C}=\mathrm{O})$ of acac was observed in FTIR spectra. That meant the chelation was good enough to synthesize a stable titania colloid when appropriate solvent was chosen.

For a comparison, Fig. 3 shows the FTIR spectra of titania colloid without chelation. The broad band at around $3400 \mathrm{~cm}^{-1}$ was assigned to stretching vibrations of $\mathrm{Ti}-\mathrm{OH}$ groups. The band at $1625 \mathrm{~cm}^{-1}$ was assigned to the bending mode of adsorbed water 


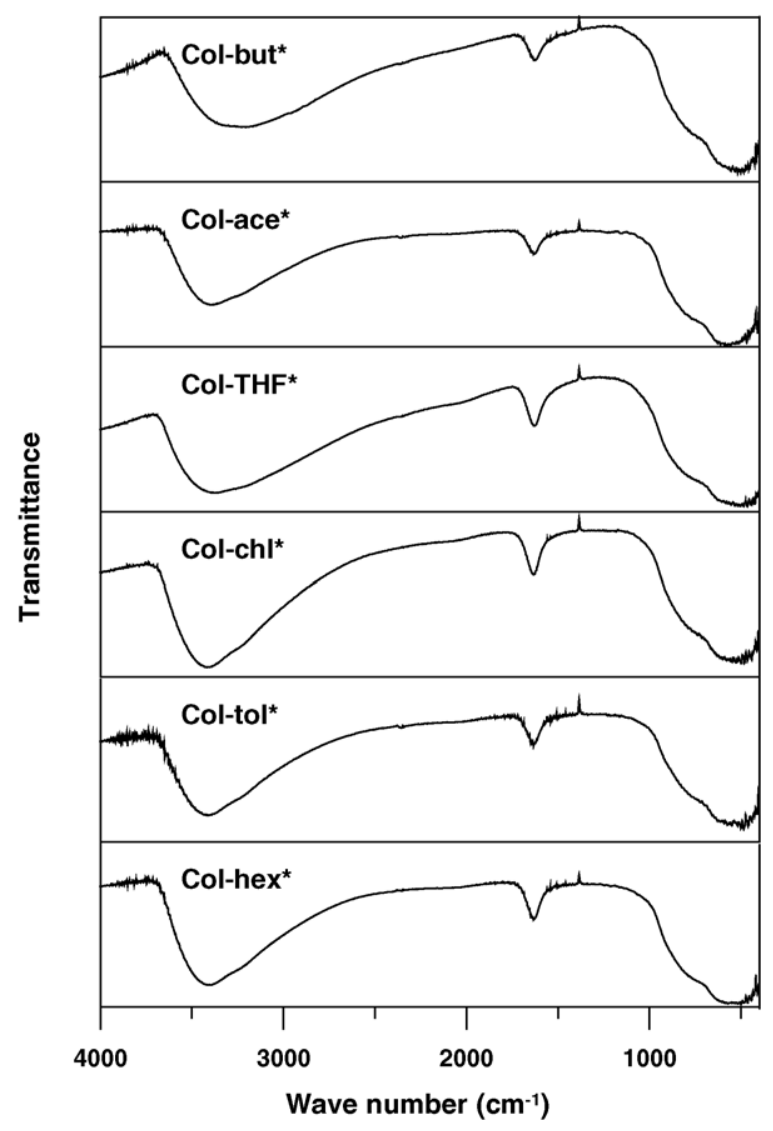

Fig. 3. FTIR spectra of titania colloid prepared by un-modified TIP.

[32]. A broad band between $400 \mathrm{~cm}^{-1}$ and $700 \mathrm{~cm}^{-1}$ should be due to the envelope of the phonon bands of Ti-O-Ti bond. No bands appeared in the range from $1580 \mathrm{~cm}^{-1}$ to $1530 \mathrm{~cm}^{-1}$. So, it could be summarized that the two bands from $1580 \mathrm{~cm}^{-1}$ to $1530 \mathrm{~cm}^{-1}$ were the characterization of chelation.

\subsection{XRD analysis}

The crystallization behavior of as-prepared and sintered titania colloid were obtained from Fig. 4. Fig. 4a depicts the XRD pattern of colloid Col-ace. There were no any crystalline characters in the Fig. 4a, which indicated that the as-prepared titania colloid was amorphous. Fig. 4a takes Col-ace as an example and the other titania colloid prepared from different solvent showed a similar amorphous form, except for Col-hex. Fig. $4 \mathrm{~b}$ is the XRD pattern of Col-hex. A small peak appeared at around $2 \theta=23.5^{\circ}$. The understanding of this small crystalline peak of Col-hex was under further studies. Fig. $4 \mathrm{c}$ shows the XRD pattern of titania colloid prepared in acetone solvent without chelation. It also showed an amorphous form. Other colloids prepared without chelation had the same amorphous structure, including that prepared in hexane. After sintering at $450^{\circ} \mathrm{C}$ for $1 \mathrm{~h}$ from room temperature at a $5{ }^{\circ} \mathrm{C} \mathrm{min}^{-1}$ heating rate, the XRD pattern of Col-ace was shown in Fig. $4 \mathrm{~d}$ and the $\mathrm{TiO}_{2}$ anatase form was observed clearly. All of the other titania colloids turn to the same anatase pattern after sintering, including Col-hex. This implied that the stable colloid would be useful in the applica-

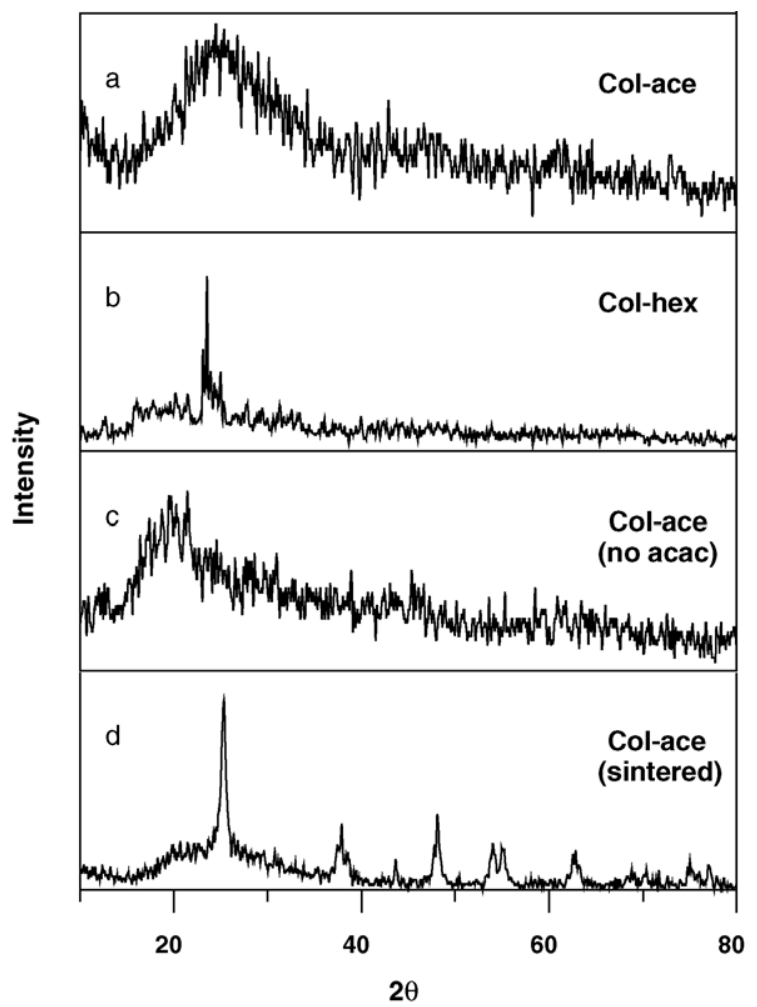

Fig. 4. XRD patterns of acac modified, un-modified, and sintered titania prepared from acetone and hexane solvent.

tion of catalysis after sintering because the anatase form of $\mathrm{TiO}_{2}$ appeared.

\subsection{Light scattering and photographs of SEM and TEM}

The particle size and aggregation phenomena of synthesized titania colloid were obtained from light scattering, SEM, and TEM observations. From the observation of experiments, the colloids of Col-THF, Col-ace, and Col-but were clear solution; Col-chl was a turbid solution; Col-tol and Col-hex showed a clear interface containing precipitation and a turbid upper solution. The particle sizes measured from light scattering were listed in Table 2, which represented the average particle size for the colloids without precipitation, and the size of the smallest particles of all for the colloids with precipitation.

SEM photographs exhibited the aggregation phenomena and particles size in dried form of colloids prepared from different solvents, as shown in Fig. 5. Fig. 5a shows the SEM photograph

Table 2

The calculated $R_{\mathrm{a}}$ and the average particle size $D$ of acac modified $\mathrm{TiO}_{2}$ colloids obtained from light scattering

\begin{tabular}{lcc}
\hline Sample & $D(\mathrm{~nm})$ & $R_{\mathrm{a}}(\mathrm{MPa})^{1 / 2}$ \\
\hline Col-THF & 2.2 & 3.13 \\
Col-ace & 4.1 & 4.68 \\
Col-but & 13.5 & 5.73 \\
Col-chl & 69 & 7.09 \\
Col-tol & 2715 & 10.87 \\
Col-hex & 15915 & 12.85 \\
\hline
\end{tabular}



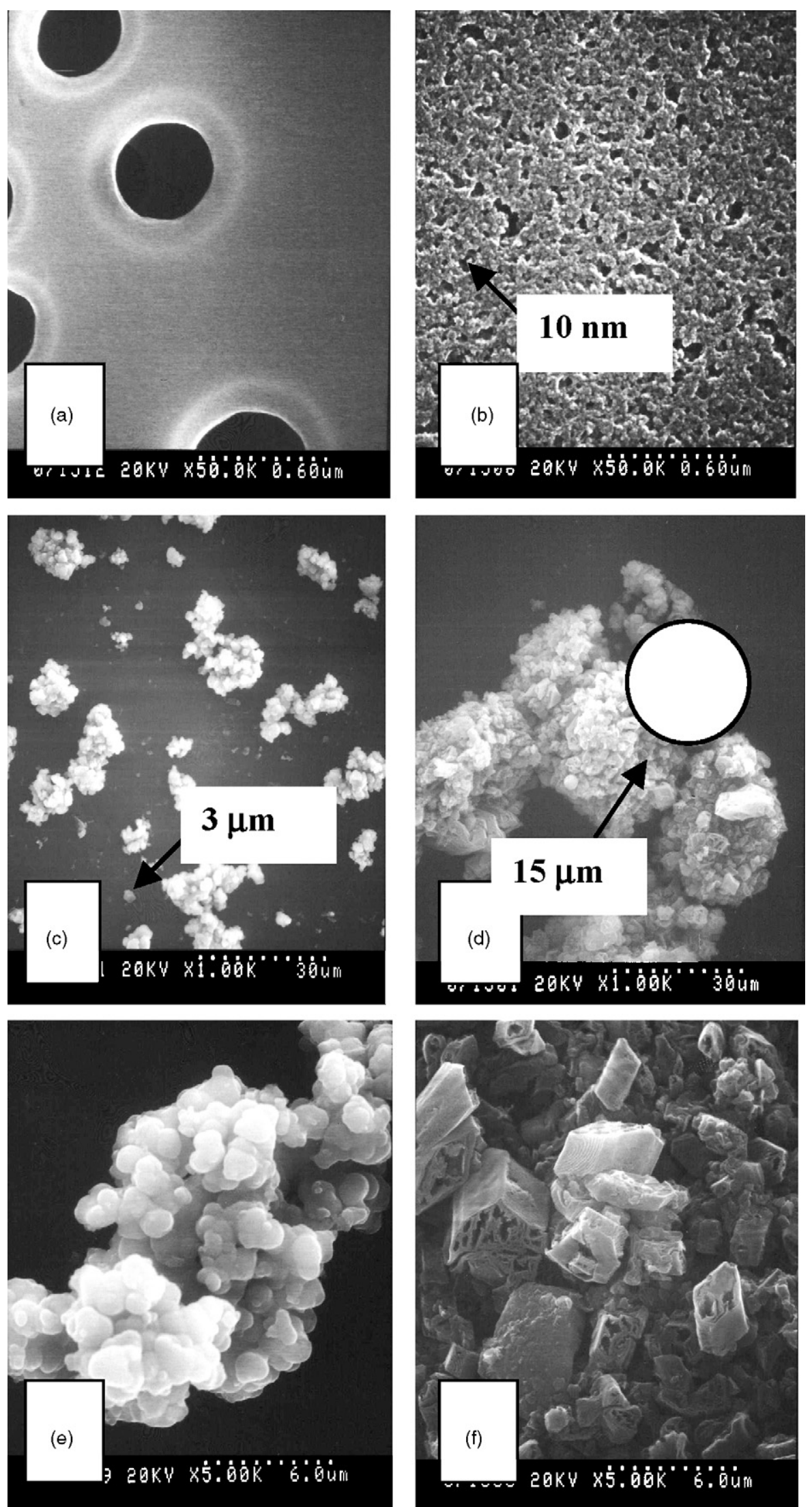

Fig. 5. SEM photographs of acac modified titania colloids prepared from (a) acetone, (b) butanol, (c) toluene, and (d) hexane solvent. The photographs of (e) and (f) were larger magnification of (c) and (d), respectively. 

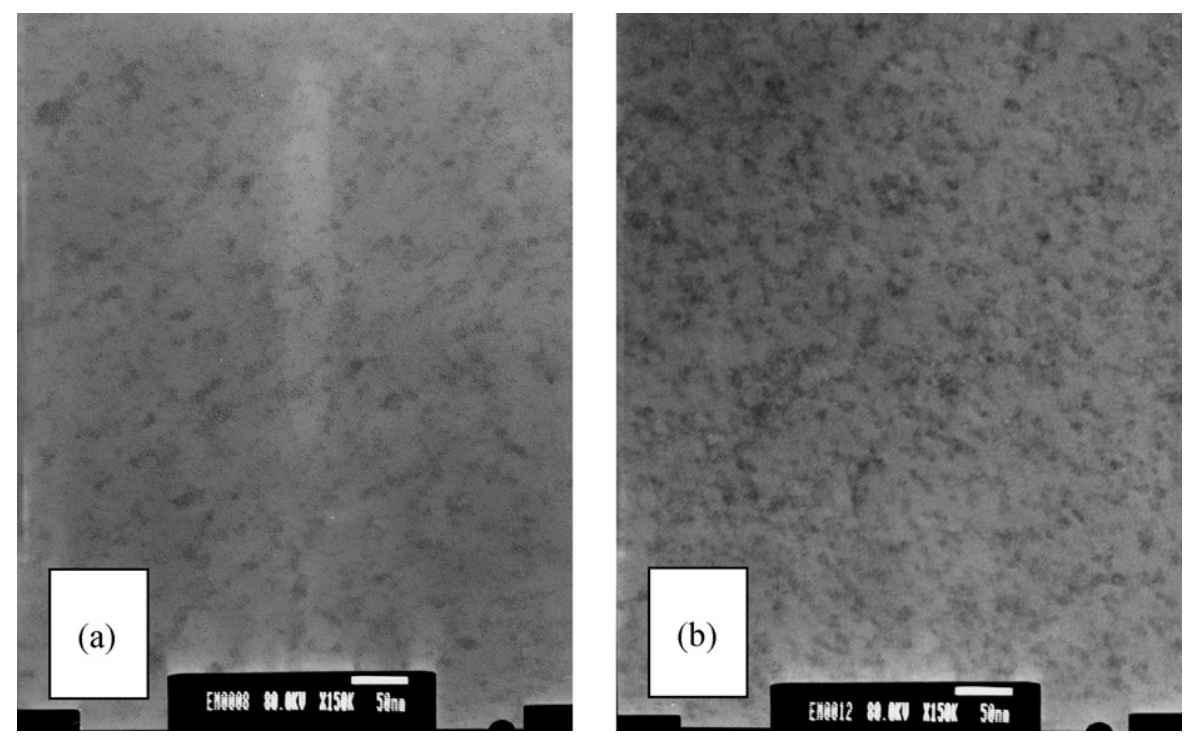

Fig. 6. TEM photographs of acac modified titania colloids prepared from (a) acetone and (b) butanol solvent.

of Col-ace. The particle size could not be clearly seen from this photograph due to the resolution limit of machine. Col-THF had the similar image, so the particle size could also not be obtained. These results stated that the particle sizes of Col-ace and Col-THF were below $10 \mathrm{~nm}$, which were in accordance with the results from light scattering. The big pores in Fig. 5a were caused by the rapid evaporation rate of acetone because of its low boiling point. Fig. 6a shows the TEM photograph of Col-ace. It showed that the particle size was lower than $10 \mathrm{~nm}$.

Fig. 5b shows the SEM photograph of Col-but. Although the sizes of particles were large enough to be identified, it was not easy to determine the average size because particles all adhered together. Nevertheless, it could be seen that particles were quite uniform in size. Roughly estimated from the arrow point in Fig. 5 b, the individual particle was about $10 \mathrm{~nm}$ in size. Fig. $6 \mathrm{~b}$ shows the TEM photograph of Col-but and the particle size was also about $10 \mathrm{~nm}$. Col-chl had a similar photograph but a larger particle size than Col-but (not shown here).

Fig. 5c and d show the SEM photographs of Col-tol and Colhex respectively. Fig. 5e and $\mathrm{f}$ were the larger magnification of Fig. 5c and d. Both of Fig. 5c and d revealed obvious aggregation, Col-hex was more significant than Col-tol. It could be claimed that the toluene and hexane were not appropriate solvent to synthesize stable titania colloids, even though the titanium precursor was previously modified with acac. The arrow point marked in Fig. 5c and d indicated the relatively small particle suspended in the upper solution respectively, which were about $3 \mu \mathrm{m}$ and $15 \mu \mathrm{m}$ in size and were in accordance with the measured results from light scattering. Notably, although both of Col-tol and Col-hex showed obvious aggregation, they had different particle shapes. The aggregated particle shape of Col-tol was fundamentally sphere; however, Col-hex displayed an irregular particle shape containing irregular pores. In the preparation of Col-tol, the solution became turbid and formed precipitation gradually in $30 \mathrm{~min}$ after the water was added. In the preparation of Col-hex, the solution had quick and clear sedimentation in $30 \mathrm{~s}$ when the water was added. It seemed that too violent aggregation of particles was responsible for the irregular shape and the porous structure.

\subsection{Hansen solubility parameter}

In the FTIR section, it demonstrated that all TIP in different organic solvents were chelated with acac. If TIP was not chelated with acac for a starting material, all of TIP would become titania through a very rapid sol-gel reaction when water was added into the solution and then precipitated fast. However, even the TIP was chelated with acac before the addition of water, the titania colloid had different stability when the sol-gel reaction of TIP was proceeded in different organic solvents. In this research, the Hansen solubility parameters (HSP) were used to explain different dispersion phenomena of titania colloids prepared in different organic solvents and tried to find appropriate HSP of acac modified titania to fit the experimental data.

Hildebrand and Scott [33] were the first ones to use the term of solubility parameter. The Hildebrand solubility parameter was defined as the square root of the cohesive energy density:

$\delta=\left(\frac{E}{V}\right)^{1 / 2}\left(\mathrm{MPa}^{1 / 2}\right)$

$E$ was the energy of vaporization of the pure solvent, and $V$ was its molar volume. However, the drawback of the Hildebrand solubility parameter was that the approach was only limited to a regular solution, and did not account for association between molecules, such as those incorporated with polar and hydrogen bonding interaction. A widely used solubility parameter approach was based on Hansen solubility parameter [34-36]. Hansen proposed an extension of the Hildebrand parameter method to polar and hydrogen bonding. The fundamental concept of HSP was that the total energy of vaporization of a liquid was divided into several individual parts, including dispersion 
energy, polar energy, and hydrogen bonding energy. These three energies were the required energies to separate liquid molecules.

So, the total vaporization energy, and thus the total cohesive energy, could be described as the following equations:

$E=E_{\mathrm{d}}+E_{\mathrm{p}}+E_{\mathrm{h}}$

$\frac{E}{V}=\frac{E_{\mathrm{d}}}{V}+\frac{E_{\mathrm{p}}}{V}+\frac{E_{\mathrm{h}}}{V}$

$\delta_{\mathrm{t}}^{2}=\delta_{\mathrm{d}}^{2}+\delta_{\mathrm{p}}^{2}+\delta_{\mathrm{h}}^{2}$

The index of $\mathrm{d}, \mathrm{p}$, and $\mathrm{h}$ meant dispersion, polar, and hydrogen bonding. The total solubility parameter, $\delta_{\mathrm{t}}$, was sometimes denoted the Hildebrand solubility parameter. $\delta_{\mathrm{d}}, \delta_{\mathrm{p}}$, and $\delta_{\mathrm{h}}$ represented dispersion or non-polar solubility parameter, polar solubility parameter, and hydrogen bonding solubility parameter, respectively; they were generally called the Hansen solubility parameters (HSP). Materials had high affinity for each other if they had similar HSP. The extent of the similarity in a given situation governed the extent of the interaction. Moreover, Hansen and Skaarup [36] developed Eq. (5) for the solubility parameter "distance", $R_{\mathrm{a}}$, between solvent and solute based on their respective partial solubility parameter components:

${ }^{i j}\left(R_{\mathrm{a}}\right)=\left[4\left({ }^{i} \delta_{\mathrm{d}}-{ }^{j} \delta_{\mathrm{d}}\right)^{2}+\left({ }^{i} \delta_{\mathrm{p}}-{ }^{j} \delta_{\mathrm{p}}\right)^{2}+\left({ }^{i} \delta_{\mathrm{h}}-{ }^{j} \delta_{\mathrm{h}}\right)^{2}\right]^{1 / 2}$

where ${ }^{i j}\left(R_{\mathrm{a}}\right)$ : solubility parameter distance of solvent $(i)$ and solute $(j)$ on three-dimensional projected map; ${ }^{i} \delta$ : Hansen parameter of solvent; ${ }^{i} \delta$ : Hansen parameter of solute.

In this study, a simple approach was utilized to estimate the HSP of solute. In our system, the solute was acac modified titania. As mentioned above, similar HSP of titania and solvent meant that the chemical nature of titania and solvent were similar and they had high affinity, which made it possible for titania to disperse well in the solvent. Based on Eq. (5), if the values of HSP for titania and solvent were similar, the calculated $R_{\mathrm{a}}$ would be small. Hence, the summation of $\left(R_{\mathrm{a}}\right)^{2}$ for those stable colloids should be minimal. According to the results of experimental, the well dispersed titania colloids were synthesized in the solvent of THF, acetone, and butanol. The $\left(R_{\mathrm{a}}\right)^{2}$ of these three stable colloids, Col-THF, Col-ace, and Col-but, were summed and set as $G$ :

$G\left({ }^{j} \delta_{\mathrm{d}},{ }^{j} \delta_{\mathrm{p}},{ }^{j} \delta_{\mathrm{h}}\right)={ }^{i j}\left(R_{\mathrm{a}}\right)_{\text {Col-THF }}^{2}+{ }^{i j}\left(R_{\mathrm{a}}\right)_{\text {Col-ace }}^{2}+{ }^{i j}\left(R_{\mathrm{a}}\right)_{\text {Col-but }}^{2}$
$G$ was a function of ${ }^{j} \delta_{\mathrm{d}},{ }^{j} \delta_{\mathrm{p}}$, and ${ }^{j} \delta_{\mathrm{h}}$, which were independent variables. The optimal values of ${ }^{j} \delta_{\mathrm{d}},{ }^{j} \delta_{\mathrm{p}}$, and ${ }^{j} \delta_{\mathrm{p}}$ to meet the requirement of minimum $G$ could be obtained from solving the following three equations, Eqs. (7)-(9).

$$
\begin{gathered}
\frac{\partial G}{\partial^{j} \delta_{\mathrm{d}}}=0 \\
\frac{\partial G}{\partial^{j} \delta_{\mathrm{p}}}=0 \\
\frac{\partial G}{\partial^{j} \delta_{\mathrm{h}}}=0
\end{gathered}
$$

And then, the optimal values of ${ }^{j} \delta_{\mathrm{d}},{ }^{j} \delta_{\mathrm{p}}$, and ${ }^{j} \delta_{\mathrm{h}}$ for titania, i.e. the solutions of Eqs. (7)-(9) were determined as:

$$
\begin{aligned}
j^{j} \delta_{\mathrm{d}} & =\frac{\left.{ }^{i} \delta_{\mathrm{d}}\right|_{\text {Col-THF }}+\left.{ }^{i} \delta_{\mathrm{d}}\right|_{\text {Col-ace }}+\left.{ }^{i} \delta_{\mathrm{d}}\right|_{\text {Col-but }}}{3} \\
{ }^{j} \delta_{\mathrm{p}} & =\frac{\left.{ }^{i} \delta_{\mathrm{p}}\right|_{\text {Col-THF }}+\left.{ }^{i} \delta_{\mathrm{p}}\right|_{\text {Col-ace }}+\left.{ }^{i} \delta_{\mathrm{p}}\right|_{\text {Col-but }}}{3} \\
{ }^{j} \delta_{\mathrm{h}} & =\frac{\left.{ }^{i} \delta_{\mathrm{h}}\right|_{\text {Col-THF }}+\left.{ }^{i} \delta_{\mathrm{h}}\right|_{\text {Col-ace }}+\left.{ }^{i} \delta_{\mathrm{h}}\right|_{\text {Col-but }}}{3}
\end{aligned}
$$

The required $\delta_{\mathrm{d}}, \delta_{\mathrm{p}}$, and $\delta_{\mathrm{h}}$ of THF, acetone, and butanol were listed in Table 3. The calculated HSP of titania from Eqs. (10) to (12) were also listed in Table 3. Based on the estimated HSP of titania, the distance $R_{\mathrm{a}}$ of titania colloids in various solvents were then calculated from Eq. (5) and listed in Table 2.

The correlation of calculated solubility parameter distance $R_{\mathrm{a}}$ and measured particle size was plotted in Fig. 7. It was shown that the solubility parameter distance $R_{\mathrm{a}}$ and particle size for stable colloid had positive correlation. In our experiments, the colloid would be stable if $R_{\mathrm{a}}$ was lower than about $6(\mathrm{MPa})^{1 / 2}$. In the range of $R_{\mathrm{a}}=6-9(\mathrm{MPa})^{1 / 2}$ roughly, the colloid would exhibit turbidity. However, if $R_{\mathrm{a}}$ was larger than $9(\mathrm{MPa})^{1 / 2}$ approximately, the colloid would have notable precipitation, such as Col-tol and Col-hex.

Finally, an additional parameter should be discussed, the molar volume. The molar volume was usually used successfully as a fourth parameter for discussion on molecular size effects. The solvents caused precipitation, such as toluene and hexane, had larger molar volume, as shown in Table 3. The Hildebrand solubility parameter theory also emphasized that solvents with smaller molar volume showed better solubility than those with larger one, even though they might have the same solubility

Table 3

$\mathrm{HSP}$ of the tested organic solvents and acac modified $\mathrm{TiO}_{2}$, plus the fourth parameter (molar volume) of the tested organic solvents

\begin{tabular}{llccc}
\hline & $\delta_{\mathrm{d}}(\mathrm{MPa})^{1 / 2}$ & $\delta_{\mathrm{p}}(\mathrm{MPa})^{1 / 2}$ & $\delta_{\mathrm{h}}(\mathrm{MPa})^{1 / 2}$ & 8 \\
\hline THF & 16.8 & 5.7 & 7 & 81.7 \\
Acetone & 15.5 & 10.4 & 15.8 & 74.0 \\
Butanol & 16 & 5.7 & 5.7 & 91.5 \\
Chloroform & 17.8 & 3.1 & 2 & 80.7 \\
Toluene & 18 & 1.4 & 0 & 106.8 \\
Hexane & 14.9 & 0 & 10.3 & -131.6 \\
Acac modified $\mathrm{TiO}_{2}$ & 16.1 & 7.3 & & - \\
\hline
\end{tabular}




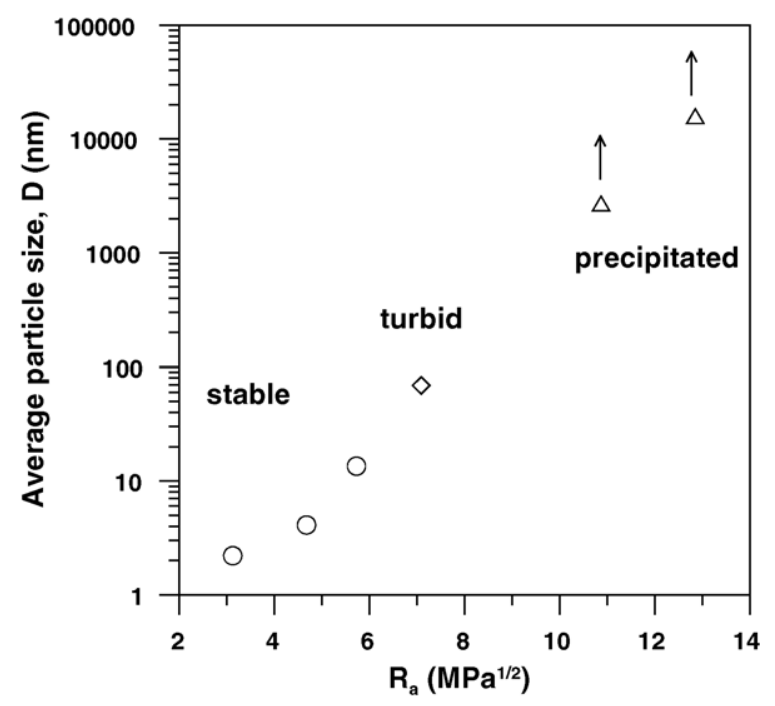

Fig. 7. Correlation between the particle size measured from light scattering and $R_{\mathrm{a}}$ estimated from HSP of acac modified titania.

parameters [33]. Efforts to qualify molar volume as a new solubility parameter had not been particularly successful.

\section{Conclusion}

The well-dispersed titania colloid could be prepared in a sol-gel reaction by using acac modified TIP as titanium precursor and selecting appropriate solvent. The FTIR measurement demonstrated that the TIP was chelated with acac, which slowed down the reaction of hydrolysis/condensation of TIP. If TIP was not chelated with acac previously, all colloid solutions would precipitate fast when the sol-gel reaction proceeded. Although the as-synthesized titania colloids were amorphous, after sintering at $4500^{\circ} \mathrm{C}$, all titania colloids showed obvious anatase form from XRD detection. From light scattering and photographs of SEM and TEM, it revealed that the chemical nature of different solvents influenced the particle size and aggregation phenomenon of titania significantly. By utilizing the concept of Hansen solubility parameter, the HSP of acac modified titania can be estimated by a simple approach. From the present experimental data, the acac modified titania colloid would be stable if the value of $R_{\mathrm{a}}$ was lower than about $6(\mathrm{MPa})^{1 / 2}$, turbid if $R_{\mathrm{a}}$ was in the range of 6-9 $(\mathrm{MPa})^{1 / 2}$ roughly, and precipitated if $R_{\mathrm{a}}$ was larger than around $9(\mathrm{MPa})^{1 / 2}$. The currently developed approach should be extendable to a wide range of various solvents, and the estimated value of $R_{\mathrm{a}}$ will provide a good prediction on whether the selected solvent is appropriate or not to prepare a well dispersed titania colloid.

\section{Acknowledgement}

The authors would like to thank the Instrumentation Center, National Taiwan University, Ching-Yen Lin, and Chih-Yuan Tang for the using of SEM.

\section{References}

[1] J.W. Yoon, T. Sasaki, N. Koshizaki, Thin Solid Films 276-282 (2005) 483.

[2] B.M. Reddy, I. Ganesh, A. Khan, J. Mol. Catal. A: Chem. 223 (2004) 295.

[3] H. Kominami, H. Kumamoto, Y. Kera, B. Ohtani, Appl. Catal. B: Environ. 30 (2001) 329.

[4] H. Ding, M.K. Ram, C. Nicolini, J. Mater. Chem. 12 (2002) 3585.

[5] B. O'Regan, M. Gratzel, Nature 353 (1991) 737.

[6] P.L. Kuo, T.C. Chang, L.M. Lu, J. Appl. Polym. Sci. 44 (1992) 859.

[7] S. Creutz, R. Jérôme, G.M.P. Kaptijn, A.W. van der Werf, J.M. Akkeman, J. Coat. Technol. 70 (1998) 41.

[8] O.Y. Uryupina, N.V. Serebryakova, V.I. Roldughin, Adv. Colloid Interface Sci. 104 (2003) 227.

[9] J. Schulz, H. Hohenberg, F. Pflücker, E. Gärtner, T. Will, S. Pfeiffer, R. Wepf, V. Wendel, H. Gers-Barlag, K.P. Wittern, Adv. Drug Deliv. Rev. 54 (2002) 157.

[10] C.E. Giacomelli, M.J. Avena, C.P.D. Pauli, Langmuir 11 (1995) 3483.

[11] M. Uchida, H.M. Kim, T. Kokubo, S. Fujibayashi, T. Nakamura, J. Biomed. Mater. Res. 64A (2003) 164.

[12] M. Navarro, M.P. Ginebra, J. Clément, S. Martínez, G. Avila, J.A. Planell, J. Am. Ceram. Soc. 86 (2003) 1345.

[13] S. Tursiloadi, H. Imai, H. Hirashima, J. Non-Cryst. Solids 350 (2004) 271.

[14] J. Sun, M. Iwasa, L. Gao, Q. Zhang, Carbon 42 (2004) 885.

[15] M. Taramasso, G. Perego, B. Notari, U.S. Patent 4,410,501, 1983.

[16] J. Whitehead, Titanium compounds, inorganic, in: M. Grayson (Ed.), KirkOthmer Encyclopaedia of Chemical Technology, vol. 23, third Edn, Wiley, New York, 1983, p. 131.

[17] K.I. Hadjiivanov, D.G. Klissurski, Chem. Soc. Rev. 25 (1996) 61 (and references therein).

[18] M.A. Vannice, R.L. Garten, J. Catal. 56 (1979) 236.

[19] G.M. El Shafei, C.A. Philip, J. Colloid Interface Sci. 176 (1995) 55.

[20] M. Malmsten, J. Colloid Interface Sci. 166 (1994) 333.

[21] M.A. Anderson, M.J. Gieselmann, Q. Xu, J. Membr. Sci. 39 (1988) 243.

[22] K.R. Rogan, A.C. Bentham, G.W.A. Beard, I.A. George, D.R. Skuse, Progr. Colloid Polym. Sci. 97 (1994) 97.

[23] L. Odberg, S. Sandberg, Langmuir 11 (1995) 2621.

[24] T.J. Trentler, T.E. Denler, J.F. Bertone, A. Agrawal, V.L. Colvin, J. Am. Chem. Soc. 121 (1999) 1613.

[25] W.B. Russel, D.A. Saville, W.R. Schowalter, Colloidal Dispersions, Cambridge University Press, Cambridge, 1989.

[26] S. Coffey (Ed.), Rodds's Chemistry of Carbon Compounds, Part D, vol. 1, Elsevier, Amsterdam, 1965, pp. 71.

[27] M.L. Eidinoff, J. Am. Chem. Soc. 67 (1945) 2072.

[28] T.W. Graham Solomons, Organic Chemistry, fifth ed., John Wiley and Sons, Inc., New York, 1992 (Chapter 17).

[29] D.L. Pavia, G.M. Lampman, G.S. Kriz, Introduction to Spectroscopy, W.B. Saunders Co., Philadelphia, London, Toronto, 1979 (Chapter 2).

[30] A. Léaustic, F. Babonneau, J. Livage, Chem. Mater. 1 (1989) 240.

[31] S.W. Boland, S.C. Pillai, W.D. Yang, S.M. Haile, J. Mater. Res. 19 (2004) 1492.

[32] W. Guo, Z. Lin, X. Wang, G. Song, Microelectron. Eng. 66 (2003) 95.

[33] J. Hildebrand, R.L. Scott, The Solubility of Nonelectrolytes, third ed., Reinhold, New York, 1950.

[34] C.M. Hansen, J. Paint Technol. 39 (1967) 104.

[35] C.M. Hansen, J. Paint Technol. 39 (1967) 505.

[36] C.M. Hansen, K. Skaarup, J. Paint Technol. 39 (1967) 511. 